\title{
Dissociative Experiences in Hypothetically Psychosis-Prone College Students
}

By: Cameron A. Pope and Thomas R. Kwapil

Pope, C.A., \& Kwapil, T.R. (2000). Dissociative experiences in hypothetically psychosis-prone college students. Journal of Nervous and Mental Disease, 188(8), 530-536.

Made available courtesy of Lippincott, Williams \& Wilkins, Inc.: http://journals.Iww.com/jonmd/Abstract/2000/08000/Dissociative Experiences in Hypothetically.9.as px

***Reprinted with permission. No further reproduction is authorized without written permission from Lippincott, Williams \& Wilkins, Inc. This version of the document is not the version of record. Figures and/or pictures may be missing from this format of the document. ***

\begin{abstract}
:
The relationship between dissociative experiences and psychosis proneness was investigated in a sample of 523 college undergraduates. Participants were administered the Dissociative Experiences Scale (DES), the Perceptual Aberration Scale, the Magical Ideation Scale, the Social Anhedonia Scale, and the Physical Anhedonia Scale. As hypothesized, the Perceptual Aberration and Magical Ideation Scales were positively correlated with the DES. The Social Anhedonia Scale had a modest correlation with the DES, but this relationship was largely mediated by the Perceptual Aberration and Magical Ideation Scales. The Physical Anhedonia Scale was uncorrelated with the DES. Exploratory factor analysis of the psychosis-proneness scales and the DES subscales resulted in a three-factor solution: dissociative experiences, positive schizotypy, and negative schizotypy. The DES depersonalization subscale loaded on both the dissociation and positive schizotypy factors.
\end{abstract}

Keywords: psychology | dissociative experiences | psychosis | dissociative experiences scale | perception aberration scale | magical ideation scale | social anhedonia scale | schizotypy

\section{Article:}

The authors are indebted to Jeremy Hinson, Michael Wrobel, and Jason Cubbin for their assistance with the data collection. 
The present study investigated the relationship between dissociative experiences and psychosis proneness in a nonclinical sample. Both the empirical and descriptive psychopathology literature have noted overlaps between the symptoms of schizophrenia and dissociative disorders. These similarities have resulted in erroneous diagnoses and in confusion concerning etiological factors associated with schizophrenia and dissociative disorders (Allen et al., 1997; Ellason and Ross, 1995; Gainer, 1994; Irwin, 1998; Modestin et al., 1996; Moise and Leichner, 1996; Ross et al., 1990b; Sandberg and Lynn, 1992; Spitzer et al., 1997). However, the relationship between dissociative and schizophrenic symptoms has not been examined in preschizophrenic or psychosis-prone young adults.

\section{Psychosis Proneness}

Current models of the etiology of schizophrenia (e.g., Andreasen, 1999; Gottesman, 1991; Meehl, 1990) assume that there are schizophrenia-prone (or more generally psychosis-prone) individuals who have a vulnerability for schizophrenia and related disorders. However, it is hypothesized that the majority of these individuals will not decompensate, although they may experience attenuated or transient symptoms of schizophrenia. These symptoms fall on a continuum from relatively healthy to subclinical deviance to schizophrenia-spectrum personality disorders to full-blown clinical psychosis. Thus, psychosis proneness may be expressed across a dynamic continuum of psychotic-like adjustment with severity contingent on the interaction of biopsychosocial factors (Gooding and Iacono, 1995). Identification of psychosis-prone individuals should facilitate the identification of relevant etiological factors and may hasten the development of prophylactic treatment interventions.

\section{Psychometric Identification of Psychosis Proneness}

Chapman Scales of Psychosis Proneness. To identify psychosis-prone young adults, Loren and Jean Chapman and their collaborators developed a series of self-report, true-false questionnaires that were intended to measure symptoms and traits characteristic of pre-schizophrenic adjustment. The development of the scales relied heavily on Meehl's (1964) description of schizotypy and Hoch and Cattell's (1959) description of pseudoneurotic schizophrenia. These measures included the Perceptual Aberration Scale (Chapman et al., 1978), the Magical Ideation Scale (Eckblad and Chapman, 1983), the Revised Social Anhedonia Scale (Eckblad et al.2), and the Physical Anhedonia Scale (Chapman et al., 1976). 
Validity of the Psychosis-Proneness Scales. Individuals with markedly elevated scores on these scales tend to show psychological and physiological deficits similar to those seen in schizophrenic patients. Chapman et al. (1994) conducted a 10-year longitudinal assessment of 534 psychosis-prone and control subjects. At the follow-up assessment, subjects identified by the Perceptual Aberration and Magical Ideation scales had higher rates of psychotic disorders than did control subjects. Kwapil (1998) reported that $24 \%$ of high scorers on the Social Anhedonia Scale exhibited schizophrenia-spectrum illnesses as compared with $1 \%$ of controls at a 10 -year follow-up, despite the fact that the groups did not differ on measures of psychopathology at the initial assessment. The Physical Anhedonia Scale has not proven to be an effective predictor of psychosis in longitudinal studies of college students. However, in cross-sectional research, high scorers on the Physical Anhedonia Scale have shown cognitive, social, and psychophysiological deficits similar to those shown by schizophrenic patients (e.g., Miller, 1986; Simons et al., 1982). Moreover, the New York High Risk Project (Erlenmeyer-Kimling, et al., 1993; Freedman et al., 1998) found that the offspring of schizophrenic patients who also had elevated scores on the Physical Anhedonia Scale were at heightened risk for psychosis and poor social adjustment.

\section{Dissociative Experiences}

Dissociation involves alterations in consciousness, memory, and identity, as well as disruptions in the perception of time and the environment (DSM-IV; American Psychiatric Association, 1994). Specific dissociative experiences include an inability to remember aspects of one's life, the experience of finding oneself in an unfamiliar situation, feelings of unreality (about self, others, or situations), feeling absorbed or completely lost in an activity, and feelings of being detached or disconnected from oneself (Ray et al., 1992). These experiences are conceptualized as existing on a continuum ranging from nonclinical conditions to pathological dissociation. Dissociative experiences are relatively common in the general population, occur equally often in male and female subjects, and tend to be reported less frequently with age (Ross et al., 1990a).

\section{Schizophrenia and Dissociation}

Schizophrenia and dissociative disorders share several common symptoms such as aberrant perceptual experiences and disruptions in reality testing. Several studies have reported considerable confusion in the diagnosis of schizophrenia and dissociative disorders (such as dissociative identity disorder), based on the presence of Schneiderian first rank symptoms (Schneider, 1959) in both disorders (Ellason and Ross, 1995; Gainer, 1994; Moise and Leichner, 1996; Ross et al., 1990b; Spitzer et al., 1997). These symptoms include auditory hallucinations, thought withdrawal/insertion, and delusional thinking (Ellason and Ross, 1995). Not surprisingly, patients with schizophrenia tend to report higher levels of dissociative experiences compared with control subjects. Furthermore, dissociative experiences occur more commonly in 
schizophrenic patients with productive or positive symptoms than in patients exhibiting deficit or negative symptoms of schizophrenia (Spitzer et al., 1997). Consistent with the findings for patients with schizophrenia, elevated rates of dissociative experiences have been reported in patients with schizotypal personality disorder (Allen et al., 1997; Modestin et al., 1996). However, the presence of dissociative experiences and their relationship to the development of clinical disorders has not been examined in nonclinical samples of psychosis-prone young adults.

Goals and Hypotheses of the Present Study

The present study examined the relationship between dissociative experiences, measured by the Dissociative Experiences Scale (DES; Bernstein et al., 1993; Bernstein and Putnam, 1986) and scores on the Chapmans' scales of psychosis proneness. It is hypothesized that scores on the Perceptual Aberration and Magical Ideation Scales will be positively correlated with the DES, based upon reports of elevated rates of dissociative symptoms in patients with positive-symptom schizophrenia and schizotypal personality disorder. Given that the Perceptual Aberration Scale assesses perceptual and somatic distortions, it is expected that it will have an especially strong relationship with the depersonalization subscale of the DES. It is hypothesized that scores on the anhedonia scales will be unrelated to or inversely correlated with scores on the DES.

\section{Methods}

\section{Participants}

Participants were 119 male and 404 female college undergraduates enrolled in General Psychology courses at the University of North Carolina at Greensboro. The ethnic composition of the sample was 74\% Caucasian, 22\% African American, and 4\% other (including Hispanic, Asian American, and Native American). Mean age of the subjects was 19.9 (SD = 3.3), with a range of 17 to 52. Male and female subjects did not differ on ethnic composition or age.

\section{Materials}

Psychosis-Proneness Scales. The Perceptual Aberration Scale contains 35 items that tap schizophrenic-like perceptual and bodily distortions. Sample items include, "I sometimes have the feeling that some parts of my body are not attached to the rest of me" [keyed true] and "My hands and feet have never seemed far away" [keyed false]. The Magical Ideation Scale is comprised of 30 items that tap a belief in implausible or invalid causality. Sample items include, "I have occasionally had the silly feeling that a TV or radio broadcaster knew I was listening to him" [keyed true] and "Numbers like 13 and 7 have no special powers" [keyed false]. The 
Revised Social Anhedonia Scale consists of 40 items that tap asociality and indifference to others. Sample items include, "Having close friends is not as important as people say" [keyed true] and "I sometimes become deeply attached to people I spend a lot of time with" [keyed false]. The Physical Anhedonia Scale includes 61 items that tap deficits in sensory and aesthetic pleasure. Sample items include, "There just are not many things that I ever really enjoyed doing" [keyed true] and "Beautiful scenery has been a great delight to me" [keyed false]. The items from the psychosis-proneness scales were intermixed along with a 13-item infrequency scale (Chapman and Chapman 3) that was designed to screen out subjects who responded in a random or "fake-bad" manner. Subjects who endorsed three or more items on the infrequency scale were not included in the subsequent analyses.

Dissociative Experiences Scale. The DES is a 28-item self-report scale that measures the frequency of dissociative experiences during the daily lives of respondents. Although these experiences are often characteristic of symptoms experienced by patients with dissociative disorders, the scale was not designed to provide specific diagnoses of dissociative disorders or related conditions. The DES contains subscales that tap three domains of dissociative experiences. The amnestic dissociation subscale consists of 10 items that tap experiences of losing time and failing to remember life events. Sample items include, "Some people have the experience of finding themselves in a place and having no idea how they got there," and "Some people find evidence that they have done things they do not remember doing" (subjects are instructed to indicate the percentage of time that they have each of the experiences). The absorption/derealizaton subscale consists of 12 items that tap experiences of being completely lost in a task or experiencing other people and events as not real. Sample items include, "Some people find that when they are watching television or a movie they become so absorbed in the story that they are unaware of other events happening around," and "Some people have the experience of not being sure whether things they remember happening really did happen or whether they just dreamed them." The depersonalization subscale consists of six items that assess feelings of unreality or being outside oneself. Sample items include, "Some people have the experience of feeling that other people, objects and the world around them are not real," and "Some people sometimes feel as if they are looking at the world through a fog so that other people and objects seem far away and unclear."

\section{Procedures}

Participants completed these measures (along with measures not used in this study) as part of departmental mass-screening sessions. The assessments lasted between 1.5 to 2 hours. A subset of participants completed the psychosis-proneness scales and the DES in different sessions. There were no differences between the scores of the subjects who completed the forms in one 
session and those who completed the measures in separate sessions. Subjects received course credit or payment for their participation.

\section{Results}

Table 1 contains descriptive statistics for each of the scales and subscales separately by gender. Scores on the psychosis-proneness scales were converted to standardized scores computed separately for men and women. Standardized scores were based upon data from 423 male and 1373 female college students enrolled at the University of North Carolina at Greensboro (Kwapil 4). Subsequent analyses were not reported separately by gender, as the findings were substantively the same when computed separately for males and females.

Table 1

\begin{tabular}{|c|c|c|c|c|c|c|}
\hline & \multicolumn{3}{|c|}{$\begin{array}{c}\text { Male } \\
(N=119)\end{array}$} & \multicolumn{3}{|c|}{$\begin{array}{c}\text { Female } \\
(N=404)\end{array}$} \\
\hline \multicolumn{7}{|l|}{ Psychosis-Proneness Scales } \\
\hline Magical Ideation & 10.2 & 6.3 & .87 & 9.8 & 5.9 & .85 \\
\hline Social Anhedonia & 9.8 & 5.8 & .82 & 7.6 & 5.2 & .82 \\
\hline Physical Anhedonia & 15.1 & 7.6 & .83 & 12.0 & 6.6 & .82 \\
\hline DES Absorption & 22.7 & 16.0 & .90 & 22.3 & 15.9 & .91 \\
\hline DES Amnestic & 9.7 & 10.5 & .87 & 9.8 & 9.3 & .81 \\
\hline DES Depersonalization & 5.6 & 10.6 & .86 & 5.2 & 9.9 & .84 \\
\hline
\end{tabular}

TABLE 1 Descriptive Data for the Psychosis-Proneness Scales and the Dissociative Experiences Scale

\section{Intercorrelations of the Measures}

Interrelationships of the Psychosis-Proneness Scales. Table 2 contains the zero-order correlations between scores on the psychosis-proneness scales and the DES (including the DES subscales). Given the large number of correlations computed (and the large sample size), alpha was set at .001. Consistent with previous findings (Chapman et al., 1982), the Perceptual Aberration and Magical Ideation Scales were significantly correlated, as were the two anhedonia scales. The Physical Anhedonia Scale was uncorrelated with the Perceptual Aberration and Magical Ideation Scales, whereas the Social Anhedonia Scale had modest, but significant, correlations with both of the scales. As expected, the DES subscales were highly intercorrelated. 
Table 2

\begin{tabular}{|c|c|c|c|c|c|c|c|}
\hline & \multirow[b]{2}{*}{$\mathrm{PerAb}^{b}$} & \multirow[b]{2}{*}{ MagId } & \multirow[b]{2}{*}{ PhyAnh } & \multirow[b]{2}{*}{ SocAnh } & \multicolumn{3}{|c|}{ DES $^{a}$} \\
\hline & & & & & Total & ABS & $\mathrm{AM}$ \\
\hline Magical Ideation (MagId) & $.75 *$ & & & & & & \\
\hline Physical Anhedonia (PhyAnh) & .04 & -.10 & & & & & \\
\hline Social Anhedonia (SocAnh) & $.32 *$ & $.21^{*}$ & $.43^{*}$ & & & & \\
\hline DES Total & $.43^{*}$ & $.44^{*}$ & .05 & $.24^{*}$ & & & \\
\hline DES Absorption (ABS) & $.40^{*}$ & $.41^{*}$ & .05 & $.22^{*}$ & $.96^{*}$ & & \\
\hline DES Amnestic (AM) & $.30 *$ & $.35^{*}$ & .06 & $.19 \%$ & $.88 *$ & $.76^{*}$ & \\
\hline DES Depersonalization (DEP) & $.52 *$ & $.45^{*}$ & .01 & $.26^{*}$ & $.76^{*}$ & $.63^{*}$ & $.59^{*}$ \\
\hline
\end{tabular}

Intercorrelations among the Psychosis-Proneness Scales and the DES. As hypothesized, the Perceptual Aberration and Magical Ideation Scales were moderately correlated with the DES total and subscale scores. Likewise, the Social Anhedonia Scale demonstrated a modest relationship with the DES. The Physical Anhedonia Scale did not correlate significantly with any of the DES scores.

Multiple Regression Analyses. To account more fully for the shared variance of the psychosisproneness scales and the DES, hierarchical multiple regression analyses were conducted. Table 3 shows the percentage of variance accounted for in the DES scales by the psychosis-proneness scales. The entry of the variables into the regression equations was based upon a priori hypotheses about the relationship between the two sets of measures. Scores on the Perceptual Aberration and Magical Ideation Scales were entered jointly at the first step, given the high percentage of shared variance between the two scales and the hypotheses that these measures would be associated with dissociative experiences. The Social Anhedonia and Physical Anhedonia Scales were entered at the second and third step, respectively. In each case, the Perceptual Aberration and Magical Ideation Scales accounted for a significant proportion of the variance. Neither of the anhedonia scales accounted for a significant increment in the variance of the DES total score or subscales, after the first two psychosis-proneness scales were entered.

Table 3 


\begin{tabular}{lllll}
\hline & \multicolumn{2}{c}{ DES Total } & \multicolumn{2}{c}{ DES Absorption } \\
\hline Step 1: & PerMag $^{a}$ & $.218^{*}$ & PerMag & $.186^{*}$ \\
Step 2: & SocAnh $^{b}$ & .013 & SocAnh & .011 \\
Step 3: & PhyAnh $^{c}$ & .000 & PhyAnh & .001 \\
& \multicolumn{2}{c}{ DES Amnestic } & DES Depersonalization \\
\hline Step 1: & PerMag & $.123^{*}$ & PerMag & $.283^{*}$ \\
Step 2: & SocAnh & .012 & SocAnh & .010 \\
Step 3: & PhyAnh & .002 & PhyAnh & .002 \\
\hline
\end{tabular}

${ }^{a}$ Perceptual Aberration and Magical Ideation Scales.

${ }^{b}$ Social Anhedonia Scale.

${ }^{c}$ Physical Anhedonia Scale.

${ }^{*} p<.001$.

TABLE 3 Increment in R2 in the DES due to the Psychosis-Proneness Scales

Exploratory Factor Analysis of the Psychosis-Proneness Scales and the DES Domain Scores. To further examine the relationships of the measures, an exploratory factor analysis with Varivax rotation was performed on the four psychosis-proneness scales and the three DES subscales. Using scree criterion, three factors were extracted that accounted for $81 \%$ of the variance. Table 4 contains the rotated factor loadings and percentage of variance accounted for by the three factors. The first component appeared to measure a pure dissociative symptom factor, whereas the second factor tapped positive schizotypy (but was associated with DES Depersonalization). The final component was a negative schizotypy factor that was unrelated to dissociation.

Table 4 


\begin{tabular}{lccc}
\hline & \multicolumn{3}{c}{ Factors $^{a}$} \\
\cline { 2 - 4 } & 1 & 2 & 3 \\
\hline Perceptual Aberration Scale & & .896 & \\
Magical Ideation Scale & & .885 & .879 \\
Physical Anhedonia Scale & & & .796 \\
Social Anhedonia Scale & & & \\
DES Absorption & .892 & & \\
DES Amnestic & .916 & & \\
DES Depersonalization & .716 & .422 & \\
\% of variance accounted & $32 \%$ & $28 \%$ & $21 \%$ \\
\hline
\end{tabular}

${ }^{a}$ Factor loadings less .30 were omitted from the table.

TABLE 4 Rotated 3-Factor Solution for the Psychosis-Proneness Scales and DES

\section{Discussion}

Perceptual Aberration, Magical Ideation, and Dissociative Experiences

As hypothesized, ratings on the DES were positively correlated with scores on the Perceptual Aberration and Magical Ideation Scales. This is consistent with the findings that patients with schizophrenia (especially those exhibiting positive symptoms) and schizotypal personality disorder tend to report elevated rates of dissociative experiences (e.g., Spitzer et al., 1997). The Perceptual Aberration Scale had an especially strong relationship with the DES depersonalization subscale. In general, however, the Perceptual Aberration and Magical Ideation Scales demonstrated a similar pattern of relationships with the DES subscales.

The positive correlations between the positive schizotypy scales and the DES are consistent with the overlap in the domains and the symptoms that they assess. The Perceptual Aberration Scale and the depersonalization subscale both inquire about alterations in perception and the way in which one's body is experienced. For example, the former includes the items, "At times I have wondered if my body was really my own," and "Now and then, when I look in the mirror, my face seems quite different than usual," whereas the DES includes the items, "Some people have the experience of feeling that their body does not seem to belong to them," and "Some people have the experience of looking in a mirror and not recognizing themselves." The specific content of the Magical Ideation Scale does not overlap as closely with the DES. However, both scales may tap the experience of unusual experiences and the willingness to report such experiences. 
Using the five-factor model, McCrae and Costa (1997) suggested that dissociative tendencies, aberrant perceptions, eccentric behavior, and magical thinking are characterized by elevations on the openness to experience factor-and specifically on the fantasy facet of that domain. High scores on the fantasy facet of openness are associated with an active fantasy life and such individuals may prefer the inner, imaginary world to the external world (Costa and McCrae, 1992). This is consistent with findings that the Perceptual Aberration and Magical Ideation Scales are positively correlated with the NEO-PI-R (Costa and McCrae, 1992) openness domain and the fantasy facet scores (Kwapil 4).

\section{Anhedonia and Dissociative Experiences}

The anhedonia scales demonstrated a divergent pattern of relationships with the other measures in the study. Consistent with previous findings, the Physical Anhedonia Scale had a moderate positive correlation with the Social Anhedonia Scale but was uncorrelated with the Perceptual Aberration and Magical Ideation Scales. As hypothesized, the Physical Anhedonia Scale was uncorrelated with the DES total and subscale scores. This was not surprising, given that the Physical Anhedonia Scale assesses a disinterest in sensory and bodily experiences, whereas the DES assesses unusual forms of such experiences. Consistent with McCrae and Costa's hypothesis regarding openness to experience, Kwapil 4 found that the Physical Anhedonia Scale was negatively correlated with openness (and the fantasy facet).

Contrary to the findings for the Physical Anhedonia Scale, the Social Anhedonia Scale was significantly correlated with the Perceptual Aberration and Magical Ideation Scales, and with all of the DES ratings. However, the modest zero-order correlations between the Social Anhedonia Scale and the DES appeared to be largely mediated by the effects of the Perceptual Aberration and Magical Ideation Scales. Social anhedonia did not account for a significant increment in the variance after the variance from the positive schizotypy scales was removed.

\section{Factor Structure of Psychosis Proneness and Dissociation}

The factor analysis extracted dissociative experience, positive schizotypy, and negative schizotypy factors. The relative robustness of the psychosis-proneness scales and the DES was evidenced by the fact that these measures generally did not decompose in the factor analysis. The positive and negative schizotypy factors appear consistent with positive and negative distinctions reported in schizophrenia (e.g., Andreasen, 1990; Crow, 1980) and schizotypal personality disorder (e.g., Kelley and Coursey, 1992; Kendler et al., 1991). Despite the heightened correlations between the DES subscales and the positive schizotypy scales, the respective 
measures diverged into separate factors. The only exception was the depersonalization subscale, which loaded on both the dissociation factor and the positive schizotypy factor.

\section{Applications of the Present Findings}

The present study provided information regarding the relationship between psychosis-proneness and dissociative experiences in a college-student (nonclinical) sample. However, it does not provide information about whether self-report of such experiences predicts the presence of or the risk for psychopathology. The finding of the hypothesized positive correlation of the Perceptual Aberration and Magical Ideation Scales with the DES raises the questions of whether: a) dissociative experiences are actually part of the preschizophrenic condition (and may in fact improve our identification of psychosis-prone young adults); b) dissociative experiences are not part of the preschizophrenic condition and individuals who experience dissociative experiences are actually misidentified as psychosis prone by the Perceptual Aberration and Magical Ideation Scales (in which case they add error variance to our studies of psychosis proneness); and c) dissociative experiences co-occur with psychosis-proneness but are not predictive of risk for schizophrenia or related conditions.

Obviously, further study is required to disentangle these possible explanations. Subsequent cross sectional and longitudinal studies will examine the degree to which dissociative experiences

predict or potentiate the development of psychopathology in a sample of psychosis-prone young adults and control subjects.

\section{References:}

Allen JG, Coyne L, Console DA (1997) Dissociative detachment relates to psychotic symptoms and personality decompensation. Comp Psychiatry 38:327-334.

American Psychiatric Association (1994) Diagnostic and statistical manual of mental disorders (4th ed). Washington, DC: Author.

Andreasen NC (1999) A unitary model of schizophrenia: Bleuler's "fragmented phrene" as schizencephaly. Arch Gen Psychiatry 56:781-787. 
Andreasen NC (1990) Positive and negative symptoms: Historical and conceptual aspects. Mod Prob Pharmacopsychiatry 24:1-42.

Bernstein EM, Putnam FW (1986) Development, reliability, and validity of a dissociation scale. J Nerv Ment Dis 174:727-735.

Bernstein EM, Carlson E, Putnam FW (1993) An update of The Dissociative Experiences Scale. Dissociation 11:16-25.

Chapman LJ, Chapman JP, Kwapil TR, Eckblad M, Zinser MC (1994) Putatively psychosisprone subjects 10 years later. J Abnorm Psychol 103:171-183.

Chapman LJ, Chapman JP, Miller EN (1982) Reliabilities and intercorrelations of eight measures of proneness to psychosis. J Consult Clin Psychol 50:187-195.

Chapman LJ, Chapman JP, Raulin ML (1976) Scales for physical and social anhedonia. J Abnorm Psychol 85:374-382.

Chapman LJ, Chapman JP, Raulin ML (1978) Body-image aberration in schizophrenia. J Abnorm Psychol 87:399-407.

Costa PT Jr, McCrae RR (1992) The Revised NEO Personality Inventory (NEO-PI-R and NEO Five-Factor Inventory (NEO-FFI) professional manual. Odessa, FL: Psychological Assessment Resources.

Crow TJ (1980) Molecular pathology of schizophrenia: More than one disease process? Br Med J 280:1-9. 
Eckblad M, Chapman LJ (1983) Magical ideation as an indicator of schizotypy. J Consult Clin Psychol 51:215-225.

Ellason JW, Ross CA (1995) Positive and negative symptoms in dissociative identity disorder and schizophrenia: A comparative analysis. J Nerv Ment Dis 183:236-240.

Erlenmeyer-Kimling L, Cornblatt BA, Rock D, Roberts S, Bell M, West A (1993) The New York High-Risk Project: Anhedonia, attentional deviance, and psychopathology. Schizophr Bull 19:141-153.

Freedman LR, Rock D, Roberts SA, Cornblatt BA, Erlenmeyer-Kimling L (1998) The New York High-Risk Project: Attention, anhedonia, and social outcome. Schizophr Res 30:1-9.

Gainer K (1994) Dissociation and schizophrenia: A historical review of conceptual development and relevant treatment approaches. Dissociation 7:261-271.

Gooding DC, Iacono WG (1995) Schizophrenia through the lens of a developmental psychopathology perspective. In D Cicchetti, DJ Cohen (Eds), Manual of developmental psychopathology: Risk, disorder, and adaptation (Vol II, pp 535-580). New York: Wiley.

Gottesman II (1991) Schizophrenia genesis: The origins of madness. San Francisco: Freeman.

Hoch PH, Cattell JP (1959) The diagnosis of pseudoneurotic schizophrenia. Psychiatr Q 33:1743.

Irwin HJ (1998) Dissociative tendencies and the sitting duck: Are self-reports of dissociation and victimization symptomatic of neuroticism. J Clin Psychol 54:1005-1015. 
Kelley MP, Coursey RD (1992) Factor structures of schizotypy scales. Pers Individ Diff 13:723731.

Kendler KS, Ochs AL, Gorman AM, Hewitt JK, Ross DE, Mirsky AF (1991) The structure of schizotypy: A pilot multitrait twin study. Psychiatry Res 36:19-36.

Kwapil TR (1998) Social anhedonia as a predictor of the development of schizophrenia-spectrum disorders. J Abnorm Psychol 107:558-565.

McCrae RR, Costa PT Jr (1997) Conceptions and correlates of openness to experience. In R Hogan, JA Johnson (Eds), Handbook of personality psychology. San Diego: Academic Press.

Meehl PE (1964) Manual for use with checklist of schizotypic signs (Report no. PR- 73-5). Minneapolis: University of Minnesota, Research Laboratories of the Department of Psychiatry.

Meehl PE (1990) Toward an integrated theory of schizotaxia, schizotypy, and schizophrenia. J Pers Disord 4:1-99.

Miller GA (1986) Information processing deficits in anhedonia and perceptual aberration: A psychophysiological analysis. Biol Psychiatry 21:100-115.

Modestin J, Gerhard E, Junghan M, Erni T (1996) Dissociative experiences and dissociative disorders in acute psychiatric inpatients. Comp Psychiatry 37:355-361.

Moise J, Leichner P (1996) Prevalence of dissociative symptoms and disorders within an adult outpatient population with schizophrenia. Dissociation 9:190-196.

Ray WJ, June K, Turaj K, Lundy R (1992) Dissociative experiences in a college age population: A factor analytic study of two dissociative scales. Pers Individ Diff 13:417-424. 
Ross CA, Joshi S, Currie R (1990a) Dissociative experiences in the general population. Am J Psychiatry 147:1547-1552.

Ross CA, Miller SD, Reagor P, Bjornson L, Fraser GA, Anderson G (1990b) Schneiderian symptoms in multiple personality disorder and schizophrenia. Comp Psychiatry 31:111-118.

Sandberg DA, Lynn SJ (1992) Dissociative experiences, psychopathology and adjustment, and child and adolescent maltreatment in female college students. J Abnorm Psychol 101:717-723.

Schneider K (1959) Clinical psychopathology (trans. MW Hamilton). New York: Grune and Stratton.

Simons RF, MacMillan FW, Ireland FB (1982) Reaction-time crossover in preselected schizotypic subjects. J Abnorm Psychol 91:414-419.

Spitzer C, Haug HJ, Freyberger HJ (1997) Dissociative symptoms in schizophrenic patients with positive and negative symptoms. Psychopathology 30:67-75.

2 Eckblad M, Chapman LJ, Chapman JP, Mishlove M (1982) The Revised Social Anhedonia Scale. Unpublished test (copies available from TR Kwapil, Department of Psychology, University of North Carolina-Greensboro, Greensboro, NC 27402-6164).

3 Chapman LJ, Chapman JP (1983) Infrequency Scale. Unpublished test (copies available from TR Kwapil, Department of Psychology, University of North Carolina-Greensboro, Greensboro, NC 27402-6164).

4 Kwapil TR (submitted for publication) The five-factor structure of the Chapmans' Scales of Psychosis Proneness. 